\title{
Study of childhood renal tumours using antisera to fibronectin, laminin, and epithelial membrane antigen
}

\author{
S KUMAR, T CARR, HB MARSDEN, MC CALABUIG-CRESPO
}

From the Paediatric Oncology Department, Christie Hospital and Holt Rudium Institute, Manchester

SUMMARY Using a peroxidase-antiperoxidase staining procedure, formalin fixed paraffin embedded sections of fetal and normal kidney; benign (mesoblastic nephroma); and malignant tumours (Wilms' tumour, clear cell renal carcinoma, rhabdoid renal tumour, and bone metastasising renal tumour of childhood (BMRTC)) were examined for their reactivity with antisera to fibronectin, laminin, and epithelial membrane antigen. Mesoblastic nephroma contained fibronectin but no laminin. Most Wilms' tumours lacked both fibronectin and laminin; $50 \%$ of rhabdoid renal tumours were positive for fibronectin and laminin-rhabdoid tumours as recognised morphologically may, in fact, be two separate entities. BMRTC and clear cell renal carcinoma lacked both fibronectin and laminin. Epithelial membrane antigen was present in most of the tubular Wilms' tumour but absent in blastemal Wilms' tumours. The presence of epithelial membrane antigen in rhabdoid tumours was surprising, as histologically, this type of tumour shows no sign of epithelial differentiation. Epithelial membrane antigen antiserum stained clear cell renal carcinomas: epithelial membrane antigen is found in the distal and not the proximal tubules of fetal and normal kidneys. Thus an obvious interpretation is that clear cell renal carcinomas originate from distal rather than from proximal tubules, as has always been thought. On the basis of these results and data from other published findings some possible histogenetic origins of childhood renal tumours were proposed.

Renal tumours account for about $12 \%$ of childhood solid neoplasms. Histologically, five distinct types of kidney tumours have been identified: Wilms' tumour; mesoblastic nephroma; rhabdoid renal tumour; bone metastasising renal tumour of childhood (BMRTC); and renal cell carcinoma. These five tumours differ greatly from each other in respect of their natural history, in vitro behaviour, and, most importantly, in their prognosis. ${ }^{1-6}$ At times, however, it is difficult to make an accurate diagnosis.

Wilms' tumour or nephroblastoma is the most common of all the renal tumours and has been described as a mixed sarcomatous and epithelial tumour with blastemal elements. The proportion of these components can vary considerably, which may give a tumour a monomorphic or highly varied histological appearance. The presence of tubular differentiation is regarded as a good prognostic feature. ${ }^{5}$ Wilms' tumour, like retinoblastoma, may occur in hereditary or non-hereditary forms. When hereditary it may be associated with congenital malformations like aniridia, genitourinary abnormalities, and mental

Accepted for publication 25 July 1985 retardation. ${ }^{3}$ Several recent reports implicated a locus within a chromosome (11p13) in the formation of Wilms' tumour. Interestingly, the oncogene, ras, is thought to reside in the $11 \mathrm{pl} 5$ region. $^{78}$

Mesoblastic nephroma is a well differentiated tumour of very young infants and has an excellent prognosis. ${ }^{910}$ These days the only treatment required is nephrectomy - that is, no chemotherapy or radiotherapy. In fact, both these treatments might inadvertently have been the causes of morbidity and mortality in some children before it was possible to distinguish this tumour type from Wilms' tumour.

Rhabdoid renal tumours constitute about $2 \%$ of children's kidney tumours. The tumour is composed of large cells with single prominent nucleoli and an abundant cytoplasm. The prognosis for this neoplasm is poor. ${ }^{11}$ In 1978 another renal tumour was distinguished from Wilms' and was named BMRTC by Marsden $e t$ al $^{\mathbf{1 2}}$ in the United Kingdom and clear cell sarcoma by Beckwith and Palmer ${ }^{13}$ in the United States of America. Either the epidemiology of this tumour differs greatly in these two countries ${ }^{14}$ or else the two groups of pathologists did not always refer to the same entity. Both groups of pathologists consid- 
Table 1 Details of childhood renal tumours, normal and fetal kidneys studied

\begin{tabular}{ll}
\hline Source & No examined \\
\hline Renal tumours: & \\
Wilms' tumour & \\
(a) Tubular & 9 \\
(b) Blastemal & 6 \\
Mesoblastic nephroma & 4 \\
Bone metastasing renal tumour of childhood & 6 \\
Rhabdoid renal tumour & 6 \\
Clear cell renal carcinoma & 7 \\
Normal kidneys & 9 \\
Fetal kidneys & 5 \\
\hline
\end{tabular}

ered the prognosis of this tumour to be dismal. Although renal carcinoma is the main type of kidney tumour in adults, it rarely occurs in children. The tumour has been reported as originating from proximal renal tubules and has an unfavourable prognosis.

In this study we examined the staining reaction of the five types of renal tumour, together with normal and fetal human kidneys, using antibodies to two glycoproteins (fibronectin and laminin) and epithelial membrane antigen). The aim of the study was to investigate whether or not the staining reaction was uniform within a tumour type and how it varied among the five types of children's kidney tumour.

\section{Material and methods}

\section{TISSUES}

All tumours were biopsy specimens fixed in $10 \%$ buffered formalin, embedded in paraffin, and sectioned at a thickness of $5 \mu \mathrm{m}$. Normal kidneys from children were either biopsy or necropsy specimens obtained within 18 hours of death. Fetal kidneys were collected soon after therapeutic abortion (14-21 weeks of gestation). Both normal and fetal kidneys were fixed, paraffin embedded, and sectioned using the same procedure as that for tumours. Table 1 gives details of the tissues examined.

\section{ANTIBODIES}

Fibronectin and laminin

Rabbit antihuman fibronectin was purchased from Dakopatts (Mercia-Brocades, United Kingdom) and used at a dilution of 1:40. Laminin antibody to mouse laminin, known to cross react with human laminin, was obtained from Bethesda Research Laboratories, Cambridge, United Kingdom. It was diluted 1:40 for staining. We have previously shown that it was necessary to digest the tissue section by enzymes to show the presence of fibronectin and laminin. ${ }^{15}$ Pepsin (Sigma P7012, Dorset, United Kingdom) was used as a $0.4 \% \mathrm{wt} / \mathrm{vol}$ solution in $0.01 \mathrm{M}$ hydrochloric acid.

\section{Epithelial membrane antigen}

(AES098) antibody purchased from Sera-Lab was raised in goat immunised with defatted human cream. The antiserum after absorption, reacts specifically with normal non-squamous epithelial tissues and often more strongly with tumours originating from this tissue. Epithelial membrane antigen survives formalin fixation and paraffin embedding. The antiserum was used at a dilution of 1:500, as described by Sloane and Ormerod. ${ }^{16}$

Swine antirabbit, or rabbit antigoat sera, and rabbit or goat peroxidase antiperoxidase complexes were obtained from Dakopatts (Mercia Brocades, United Kingdom).

\section{PEROXIDASE-ANTIPEROXIDASE STAINING PROCEDURE (PAP)}

PAP staining methods were carried out in accordance with Polak and Van Noorden. ${ }^{17}$ Sections embedded in wax were deparaffinised in two changes of xylene (five minutes each). Endogenous peroxidase was inhibited by treating sections with $0.5 \%$ hydrogen peroxide in methanol. The slides were then washed three times in phosphate buffered saline $0 \cdot 1 \mathrm{M}, \mathrm{pH} 7 \cdot 4$, followed by a thorough rinse in tap water.

Sections to be stained with fibronectin and laminin antiserum were treated with pepsin for two hours and washed thrice in phosphate buffered saline. Fibronectin or laminin antibody was applied for 30 minutes at room temperature. The sections were treated with swine antirabbit immunoglobulin (1:40) for 30 minutes, washed in phosphate buffered saline, and, finally, PAP complex (1:40) was added for 30 minutes. The slides were thrice washed in phosphate buffered saline, and the colour was developed using freshly prepared diaminobenzidine (DAB) (Sigma). Sections were counterstained with haematoxylin, dehydrated, cleared, and mounted.

Epithelial membrane antigen was stained without pepsin digestion of tissue sections. After the paraffin had been removed sections were sequentially treated with normal rabbit serum (1:20) for 20 minutes, epithelial membrane antigen antibody $(1: 500)$ for 30 minutes, washed with phosphate buffered saline and rabbit antigoat serum $(1: 80)$ for 30 minutes, washed with phosphate buffered saline and goat peroxidase antiperoxidase $(1: 100)$ for 30 minutes, washed with phosphate buffered saline and treated with DAB solution for 10 minutes, then stained with haematoxylin.

\section{Results}

None of the control tissue sections showed any staining when treated with normal rabbit serum. Fisher's exact test was used to analyse the results. 
Table 2 Distribution of fibronectin, laminin, and epithelial membrane antigen in kidney tumours

\begin{tabular}{|c|c|c|c|c|c|}
\hline \multirow[t]{2}{*}{ Source of tissue } & \multicolumn{2}{|l|}{ Fibronectin } & \multicolumn{2}{|l|}{ Laminin } & \multirow{2}{*}{$\begin{array}{l}\text { Epithelial } \\
\text { membrane } \\
\text { antigen }\end{array}$} \\
\hline & Tumour cells & Tubular basement & Tumour cells & Tubular basement & \\
\hline $\begin{array}{l}\text { Wilms' tumour: } \\
\text { (a) Tubular } \\
\text { (b) Blastemal } \\
\text { Rhabdoid tumour of kidney } \\
\text { Bone metastasising renal tumour of } \\
\text { childhood } \\
\text { Mesoblastic nephroma } \\
\text { Clear cell renal carcinoma }\end{array}$ & $\begin{array}{l}0 / 9 \\
0 / 5 \\
3 / 6^{*}\end{array}$ & $\begin{array}{l}9 / 9 \\
2 / 5 \\
6 / 6\end{array}$ & $\begin{array}{l}0 / 9 \\
0 / 5 \\
3 / 6^{*}\end{array}$ & $\begin{array}{l}7 / 9 \\
2 / 5 \\
6 / 6\end{array}$ & $\begin{array}{l}5 / 6 \\
0 / 6 \\
4 / 5\end{array}$ \\
\hline
\end{tabular}

*The same three tumours stained with laminin and fibronectin.

FIBRONECTIN AND LAMININ

Pepsin treatment of tissue sections was a necessary prerequisite to show the presence of both fibronectin and laminin. There were significant differences among different renal tumour types in the numbers staining positively for fibronectin $(p<0.002)$ and laminin (p < 0.04) (Table 2).

\section{Wilms' tumour}

In tubular Wilms' strong staining of basement membrane by both fibronectin and laminin antibodies was seen. Most tumour cells (in excess of $99 \%$ ) of tubular and blastemal Wilms', however, contained no fibronectin or laminin (Fig. 1). In two of nine tubular Wilms', deposits of fibronectin were seen only in the lumens of some tubules. Sparsely scattered dots of laminin were also seen in two of five tubular Wilms' but were regarded as extracellular. In two other blastemal Wilms' deposits of fibronectin occurred around what appeared to be abortive tubular structures with no actual lumens. Interestingly, the same areas in sections stained with haematoxylin and eosin seemed to be entirely blastemal with no suggestion of tubule formation.

\section{Rhabdoid tumour}

The same three of six rhabdoid tumours studied contained both laminin and fibronectin; the three others were negative for both (Fig. 2).

\section{$B M R T C$ and clear cell renal carcinoma}

All BMRTC and clear cell renal carcinomas were negative for both fibronectin and laminin (one spindle celled renal carcinoma was positive both for fibronectin and laminin).

\section{Mesoblastic nephromas}

All four mesoblastic nephromas were positive for fibronectin and negative for laminin (Fig. 3).

\section{Normal and fetal kidneys}

Normal and fetal kidneys essentially stained similarly with fibronectin and laminin antisera (Fig. 4). The notable exceptions were tubular cells, which stained positively for these two glycoproteins in fetal kidney but were negative in normal kidney. In fetal and normal kidneys fibronectin and laminin were present in Bowman's capsule, the glomerular tuft and basal lamina of tubules, and blood vessels.

Fibrovascular stroma including basement membrane Irrespective of tumour type blood vessels in all tumours contained both fibronectin and laminin. Vascular basement membrane tended to contain both fibronectin and laminin.

\section{Epithelial membrane antigen}

The distribution of epithelial membrane antigen varied significantly in the different tumour types examined $(\mathrm{p}<0.001)$ (Table 2$)$.

\section{Wilms' tumour}

Five of six tubular, but none of the blastemal Wilms', contained epithelial membrane antigen $(p<0.01)$ (Fig. 5).

\section{Rhabdoid tumour}

Eighty per cent of rhabdoid tumours contained epithelial membrane antigen (Fig. 6).

\section{BMRTC and mesoblastic nephroma}

BMRTCs and mesoblastic nephromas failed to stain with epithelial membrane antigen antibodies.

\section{Clear cell renal carcinomas}

Almost all (five of six) renal carcinomas stained strongly for epithelial membrane antigen (Fig. 7).

\section{Normal and fetal kidneys}

The prevalence of epithelial membrane antigen in kidneys was localised to the distal tubules and Henle's loop but was absent in glomeruli, proximal tubules, and stroma both in fetal and normal kidneys (Fig. 8). 

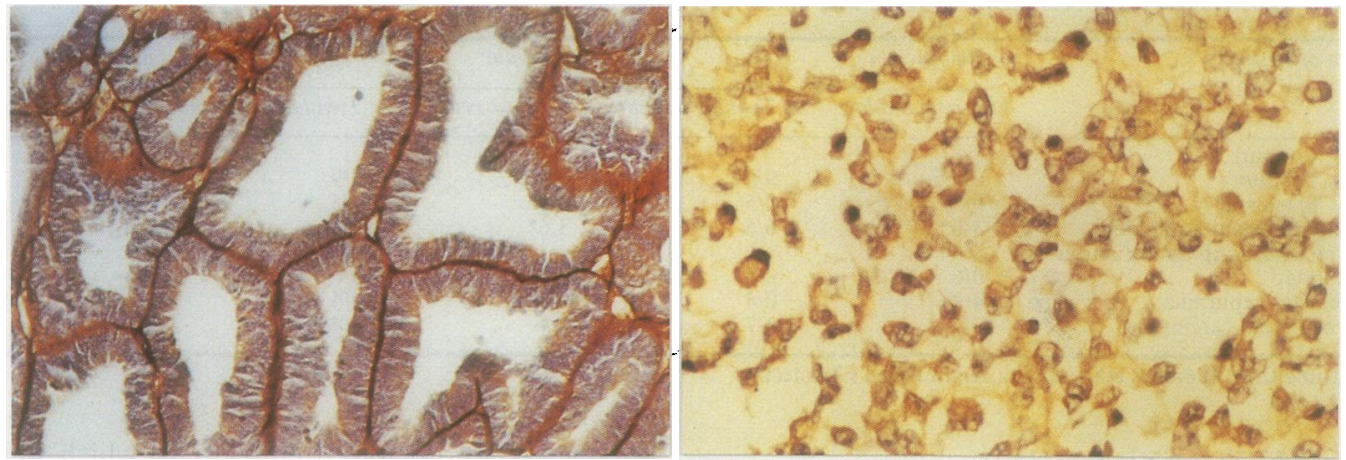

Fig. 1

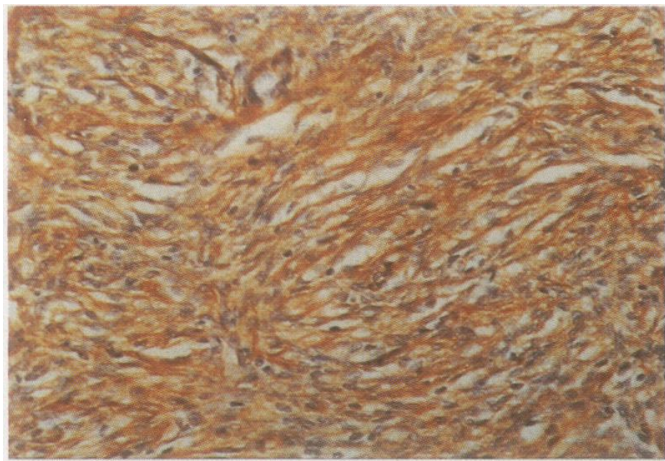

Fig. 3

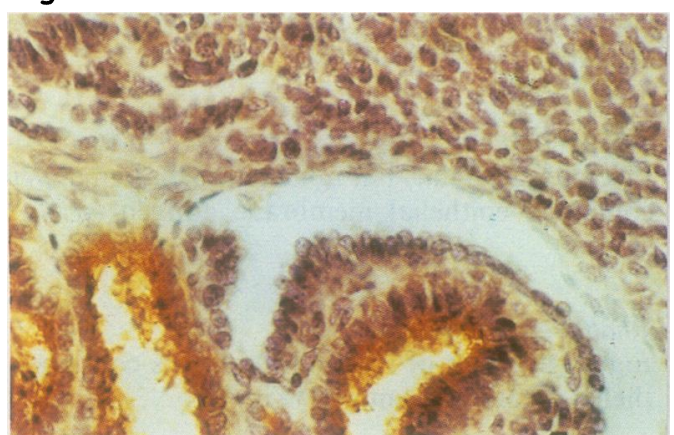

Fig. 5

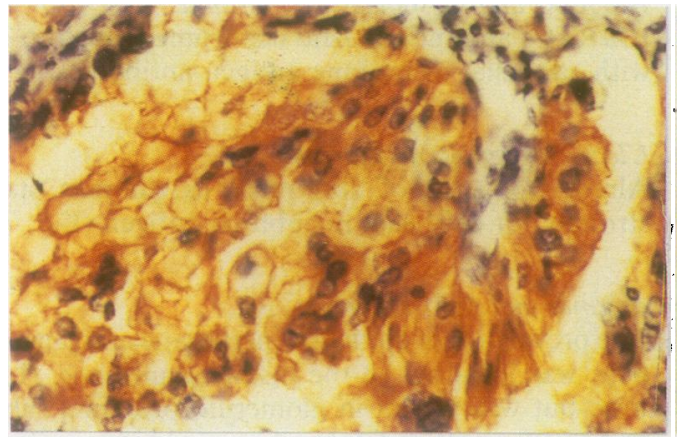

Fig.7
Fig. 2

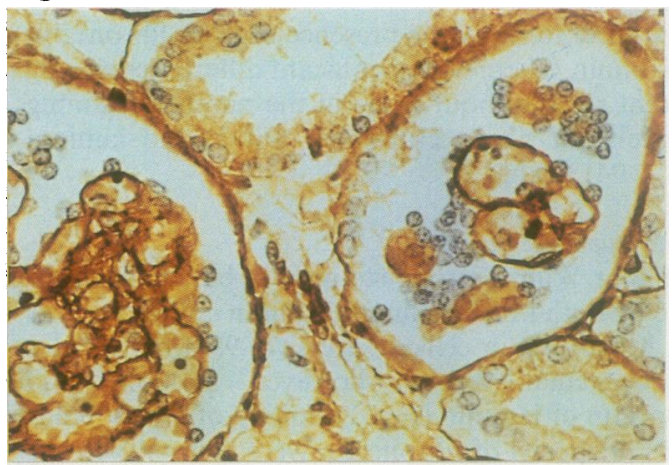

Fig. 4

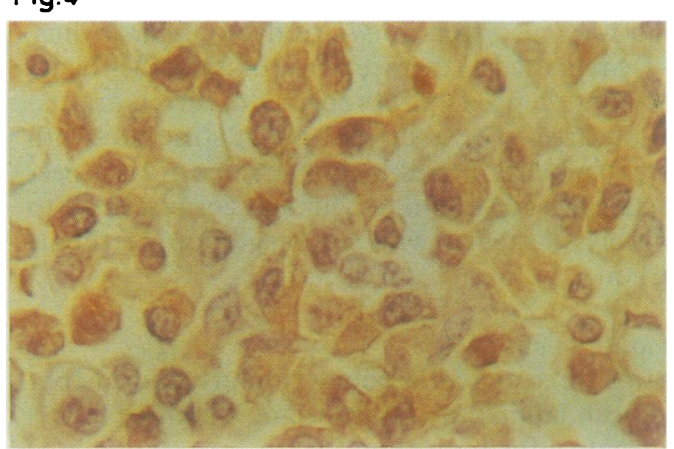

Fig. 6

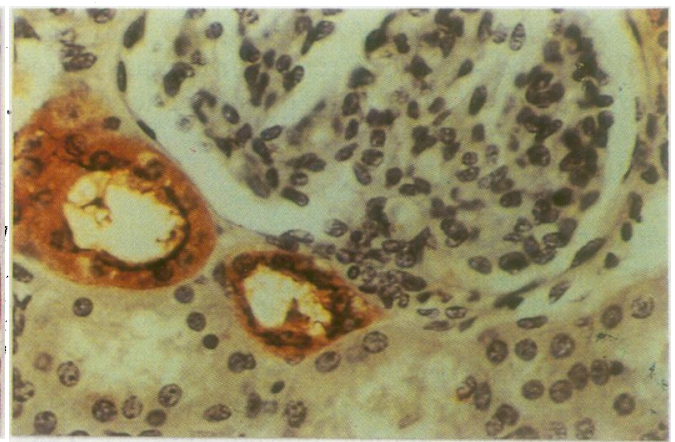

Fig. 8 
Figs. 1-8 Localisation of fibronectin, laminin, and epithelial membrane antigen using peroxidase-antiperoxidase technique.

Fig. 1 Fibronectin in completely encircled tubules in tubular Wilms'.

Fig. 2 Laminin in rhabdoid renal tumour: three of six rhabdoid tumours were positive for both laminin and fibronectin (not shown).

Fig. 3 Fibronectin in mesoblastic nephroma: all four mesoblastic nephromas contained fibronectin.

Fig. 4 Laminin in fetal kidney: laminin was seen in blood vessels, glomerular tuft, and in basal lamina of tubules.

Fig. 5 Epithelial membrane antigen in tubular Wilms': only the tubular areas of Wilms' were positive, whereas blastemal parts were negative.

Fig. 6 Epithelial membrane antigen in rhabdoid renal tumour: almost all cells stained strongly for epithelial membrane antigen.

Fig. 7 Epithelial membrane antigen in clear cell renal carcinomas: glandular structures of the tumour were clearly stained by epithelial membrane antigen antibody.

Fig. 8 Epithelial membrane antigen in normal kidneys: photomicrograph shows that distal tubules stained strongly while proximal tubules and glomeruli stained negatively.

\section{Discussion}

From the results of this study it was clear that the staining of the five types of childhood kidney tumour differed significantly both within and between histological subtypes $(p<0.05)$. These findings are important for establishing the histogenesis of renal tumours and may be of practical value to a pathologist.

During embryogenesis the development of the kidney is started by the ingrowth of the ureteric bud into the metanephrogenic mesenchyme. This, after induction by the ureteric bud, aggregates into condensates and forms epithelial tubules. ${ }^{3}$ Studies by Ekblom et al (reviewed by Ekblom ${ }^{18}$ ) on murine kidney showed that during normal nephrogenesis the acquisition and loss of fibronectin and laminin occurs in an orderly fashion. Fibronectin and laminin are

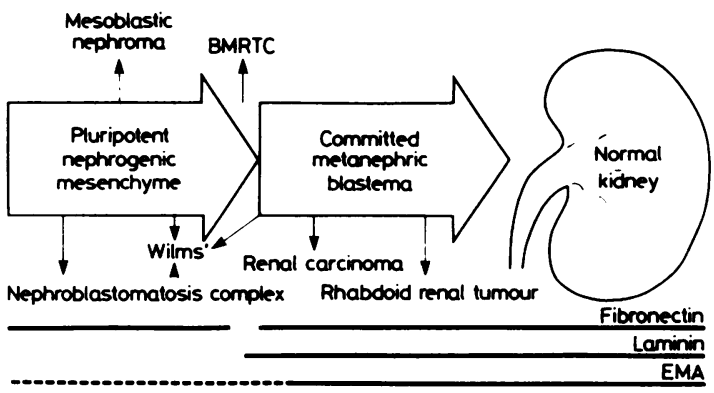

Fig. 9 Schematic representation of normal renal development and proposed histogenetic origin of childhood renal tumours. This is based on results of this study and those of Ekblom (1984), Gonzales-Crussi and Franklin's review (1984), and Sloan and Omerod's (1981) findings. Solid lines at bottom are there to show presence or absence of fibronectin, laminin, and epithelial membrane antigen during fetal development and embryogenesis. Gap in fibronectin and laminin line corresponds to induction period of renal blastema by ureteric bud. BMRTC may originate during this period.

absent in the earliest morula conceptus; fibronectin is first detected when the mesoderm appears. ${ }^{19}$ Undifferentiated and uninduced nephrogenic mesenchyme contains fibronectin and no laminin. Soon after induction fibronectin disappears and laminin becomes detectable for the first time. After the tubules have been formed their basement membrane contains both fibronectin and laminin. This orderly appearance and disappearance of the two glycoproteins could help to establish the histogenesis of kidney tumours.

It is generally believed that all kidney tumours originate from metanephric blastema. Our earlier preliminary study and the present results show that the mesoblastic nephroma contains fibronectin but lacks laminin. ${ }^{15}$ The implication is that mesoblastic nephroma probably originates from primitive nephrogenic mesenchyme rather than the committed metanephric blastema. ${ }^{20}$ In this study most of the tubular and blastemal subtypes of Wilms' tumour showed a similar distribution of fibronectin and laminin to that described by Sariola $e t$ al in a small number of kidney tumours. ${ }^{21}$ In tubular Wilms' these tubules were completely encircled by a basement membrane containing fibronectin and laminin. In contrast, none of the five blastemal tumours showed any morphological evidence of tubular differentiation, but, most interestingly, in two of these neoplasms some of the blastemal areas contained incomplete encirclement by material containing fibronectin and laminin. Tubular differentiation in Wilms' tumour indicates a good prognosis. ${ }^{5}$ Whether or not deposition of fibronectin (as seen in two cases here) relates to longer survival 
in patients with blastemal tumours needs to be investigated. The absence of laminin in blastemal Wilms' suggests that most of the blastemal cells are metanephric cells that have undergone only partial maturation. Sariola et al stated that blastemal tumour cells ".... may have lost mesenchymal phenotype but apparently did not acquire the capacity to continue differentiation by turning on the expression of basement membrane components". ${ }^{21}$ The detection in two blastemal Wilms' of material staining positively for laminin and fibronectin shows that the differentiation can proceed to a varying degree - that is, blastemal cells can have either fibronectin or laminin, or both together. The nature of factors that mediate differentiation is unknown, but transferrin is known to be important in the morphogenesis of some tissues. It has also been shown to be vital for normal nephrogenesis. ${ }^{18}$ After induction, for example, nephrogenic mesenchyme can undergo an initial burst of proliferation but for the subsequent divisions it has an absolute requirement for transferrin. Uninduced mesenchyme, however, is not responsive to transferrin.

The fact that only $50 \%$ of rhabdoid renal tumours were positive for fibronectin and laminin strongly indicates that rhabdoid tumours may not be a single entity but may include two histologically similar tumours. Differential staining needs to be related to prognosis.

The absence of fibronectin and laminin both in BMRTC and renal carcinomas (with the exception of spindle cell type) raises the possibility that these kidney tumours originate from a common ancestor that lacks both the glycoproteins. The reaction, however, with epithelial membrane antigen antibodies contradicts such a theory.

Staining with antibodies to epithelial membrane antigen clearly separated blastemal from tubular Wilms' $(p<0.01)$. Although tubular Wilms' were expected to be positive for epithelial membrane antigen, supporting experimental evidence has been lacking. ${ }^{16}$

The presence of epithelial membrane antigen (which is located in non-squamous but not squamous epithelial cells) in most of the rhabdoid tumours was surprising. ${ }^{16}$ Morphologically, this tumour is easy to diagnose, being composed of cells containing a nucleus with a single well defined nucleolus and abundant cytoplasm. The cells resemble myoblasts, but experimental evidence has not confirmed this. Vogel et al studied one rhabdoid renal tumour using a panel of monoclonal antibodies. ${ }^{11}$ Paraffin embedded sections and tissue cultured cells from this tumour were decorated by an antibody raised against a cytokeratin from human hepatoma cells. Cytokeratin is present in non-squamous and absent from squamous epithelium. The rhabdoid tumour cells, however, failed to react with an antibody made against a keratin (from stratum corneum) that was characteristic of squamous epithelium alone. It was mentioned earlier that epithelial membrane antigen is present only in non-squamous epithelium. Therefore, the staining pattern of rhabdoid tumour by epithelial membrane antigen in this study is in keeping with the reaction to cytokeratin.

The absence of epithelial membrane antigen in BMRTC and mesoblastic nephromas was predictable, as neither of these two tumour types show any morphological evidence of epithelial differentiation. Mesoblastic nephroma mostly comprises fibroblastic and myofibroblastic cells: mesoblastic nephroma is thought to originate from primitive nephrogenic mesenchyme (premetanephric), whereas the histogenesis of BMRTC remains unknown. Marsden et al ${ }^{12}$ suggested that BMRTC is probably a tumour of mesangial cells. ${ }^{12}$ A more recent paper by Haas et al doubted this conclusion. ${ }^{14}$ These authors compared the ultrastructural features of 12 such tumours with those of other renal tumours, tumours of pericytes, Schwann cells, renomedullary interstitial cells, and non-renal clear cell tumours. Although Haas et al failed to arrive at a firm histogenetic origin of BMRTC, they concluded that ultrastructurally, BMRTC resembles primitive mesenchymal cells.

A controversial finding of our investigation was the staining of clear cell renal carcinomas by epithelial membrane antigen antibodies. Epithelial membrane antigen was also present in the distal but not proximal convoluted tubules of normal and fetal kidneys. An obvious interpretation of these observations is that renal carcinoma originates from distal tubules and not, as is widely accepted, from proximal tubules: it is really remarkable that such a widely held belief is largely based on a single report published 13 years ago by Wallace and Nairn ${ }^{22}$ using two antisera, ${ }^{23}$ one of which was reactive with brush border (BB) antigen of proximal tubules and the other, Tamm-Horsfall (TH) protein, found in distal tubules and the loop of Henle. Using these antisera, Wallace and Nairn found that renal cell carcinomas contained the BB antigen but not the TH antigen. Why the results of their study and ours should differ so completely is a mystery. There may possibly be a difference between adult and childhood renal carcinomas. Lack et al, however, examined childrens' and adults' renal carcinomas and concluded that the two variants of tumour displayed similar gross and microscopic features. ${ }^{24}$ Furthermore, Lack et al stated that their pathological features suggested an origin of renal carcinoma in "distal collecting tubules (ducts of Bellini)". We also learnt from Dr S Fleming (personal communication) that in his study epithelial membrane antigen antibody stained adult renal carcinomas and distal tubules of normal 
kidneys. Therefore, the present evidence strongly suggests that the histogenesis of renal carcinoma is from distal tubules.

To conclude, Sariola's findings are the most relevant to the question of histogenesis of childhood renal tumours. ${ }^{25}$ On transplanting avascular embryonic quail kidney on to chick chorioallantoic membrane he found that the fully developed transplanted kidney's blood vessels possessed characteristic markers of the chick and not of the quail, which may indicate that some types of primary renal tumours of the kidney could have an extranephric origin.

\section{References}

${ }^{1}$ D-Angio GJ. Oncology seen through the prism of Wilms' tumor. Med Pediatr Oncol 1984;13:53-8.

${ }^{2}$ Beckwith JB. Wilms' tumor and other renal tumors of childhood. Hum Pathol 1984;14:481-92.

${ }^{3}$ Gonzalez-Crussi F. Wilms' tumor (nephroblastoma) and related renal neoplasms of childhood. Florida: CRC Press Inc, 1984.

${ }^{4}$ Kumar S, Marsden HB, Calabuig MC. Childhood kidney tumours in vitro studies and natural history. Virchows Arch (Pathol Anat) 1984;405:95-111.

${ }^{5}$ Marsden HB, Lawler W, Carr TF, Kumar S. A scoring system for Wilms' tumour: pathological study of the second Medical Research Council (MRC) trial. Int J Cancer 1984;33:365-8.

${ }^{6}$ Stambolis $C$. Benigne und potentielle maligne metanephrogene Neoplasmen: Morphologie, diagnose und klinichebedeutung. Doerr W. Leon Hardt H, eds. Stuttgart: George Thieme Verlag, 1984.

${ }^{7}$ Fisher JH, Miller YE, Sparkes RS, et al. Wilms' tumor aniridia association: segregation of affected chromosome in somatic cell hybrids; identification of cell surface antigen associated with deleted area and regional mapping of c-Ha-ras-1 oncogene, insulin gene and B-globin gene. Somatic Cell Mol Genet 1984;10: 455-64.

${ }^{8}$ Orkin SH. Wilms' tumour: molecular evidence for the role of chromosome 11. In: Rowley JD, ed. Cancer surveys: consistent chromosal aberration and oncogene in human tumours. Oxford: Oxford University Press, 1984:465-77.

${ }^{9}$ Gonzalez-Crussi F, Sotelo-Aulia C, Kidd JM. Mesenchymal renal tumours in infancy: a reappraisal. Hum Pathol 1981;12:646-57.

${ }^{10}$ Marsden HB, Lawler W. Primary renal tumours in the first year of life. Virchows Arch (Pathol Anat) 1983;399:1-9.

${ }^{11}$ Vogel AM, Gown AM, Caughlan J, Hass JE, Beckwith JB. Rhab- doid tumours of the kidney contain mesenchymal specific and epithelial specific intermediate filament proteins. Lab Invest 1984;50:232-8.

${ }^{12}$ Marsden HB, Lawler W, Kumar P. Bone-metastasizing renal tumours of childhood. Cancer 1978;42:1922-8.

${ }^{13}$ Beckwith JB, Palmer NF. Histopathology and prognosis of Wilms' tumour: results from the first national Wilms' tumour study. Cancer 1978;41:1937-48.

${ }^{14}$ Haas JE, Bonadio JF, Beckwith JB. Clear cell sarcoma of the kidney with emphasis on ultrastructural studies. Cancer 1984;54:2978-87.

${ }^{15}$ Kumar S, Marsden HB, Carr TF, Kodet R. Mesoblastic nephroma contains fibroectin but lacks laminin. J Clin Pathol 1985; 38:507-11.

${ }^{16}$ Sloane JP, Ormerod MG. Distribution of epithelial membrane antigen in normal and neoplastic tissues and its value in diagnostic tumour pathology. Cancer 1981;47:1786-95.

${ }^{17}$ Polak JM, van Noorden SV. Immunocytochemistry: practical applications in pathology and biology. Bristol: Wright PSG, 1983:11-42.

${ }^{18}$ Ekblom P. Basement membrane proteins and growth factors in kidney differentiation. In: RL Trelstad, ed. The role of extracellular matrix in development. New York: Alan $\mathbf{R}$ Liss, 1984:173-206.

${ }^{19}$ Gonzalez-Crussi F, Franklin WA. Embryonic considerations. In: Gonzalez-Crussi F, ed. Wilms' tumour (nephroblastoma) and related renal neoplasms of childhood. Florida: CRC Press, 1984:8-18.

${ }^{20}$ Snyder HM, Lack EE, Chetty-Baktavizian A, Bauer SB, Colodny AH, Retik AB. Congenital mesoblastic nephroma: relationship to other renal tumours of infancy. J Urology 1981;126:513-6.

${ }^{21}$ Sariola H, Ekblom P, Rapola J, Vaheri A, Timpl R. Extracellular matrix and epithelial differentiation of Wilms' tumour. Am J Pathol 1985;118:96-107.

${ }^{22}$ Wallace AC, Nairn RC. Renal tubular antigens in kidney tumours. Cancer 1972;29:977-81.

${ }^{23}$ Porter KA. The kidneys. In: Symmers WStC, ed. Systematic pathology. Edinburgh: Churchill-Livingston, 1978:1476.

${ }^{24}$ Lack EE, Sallan SE, Cassady JR. Renal cell carcinoma in childhood and adolescence: a clinical and pathologic study of 17 cases. Am J Path 1985;83:395.

${ }^{25}$ Sariola H. Angiogenesis of the kidney. Helsinki, Finland. University of Finland, 1985:1-40. (Thesis.)

Requests for reprints to: Dr S Kumar, Paediatric Oncology Department, Christie Hospital and Holt Rudium Institute, Wilmslow Road, Withington, Manchester M20 9BX, England. 